\title{
Catheter Insertion via Extracorporeal Membrane Oxygenation Cannula during Transcatheter Aortic Valve Implantation
}

\author{
Elmar W. Kuhn ${ }^{1} \quad$ Navid Madershahian ${ }^{1} \quad$ Tanja K. Rudolph $^{2}$ Maximilian Scherner ${ }^{1} \quad$ Yeong-Hoon Choi $^{1}$ \\ Thorsten Wahlers ${ }^{1}$
}

${ }^{1}$ Department of Cardiothoracic Surgery, Heart Center Cologne, Cologne, Germany

2 Department of Cardiology, Heart Center Cologne, Cologne, Germany

Address for correspondence Elmar W. Kuhn, MD, Department of Cardiothoracic Surgery, Heart Center Cologne, Kerpener Str. 62 50937, Cologne 50937, Germany (e-mail: elmar.kuhn@uk-koeln.de).

Thorac Cardiovasc Surg Rep 2016;5:41-43.

\begin{abstract}
Keywords

- aortic valve

- extracorporeal circulation

- transcatheter valve implantation

Background Patients referred to transcatheter aortic valve implantation (TAVI) present with multiple risk factors for intraprocedural complications.

Case Description We describe a TAVI procedure with sudden hemodynamic collapse and cardiopulmonary resuscitation. Therefore, extracorporeal membrane oxygenation cannulas were immediately implanted via vessels of the groin. A Y-connector was inserted into the arterial line of the extracorporeal membrane oxygenator using an additional sheath facilitating to insert a pigtail catheter to guide the further valve implantation.

Conclusion This case report provides an option to insert a pigtail catheter after implantation of extracorporeal membrane oxygenator cannulas without need for additional vascular access.
\end{abstract}

\section{Introduction}

Patients undergoing transcatheter aortic valve implantation (TAVI) present with multiple comorbidities such as coronary artery disease, chronic obstructive pulmonary disease, and impaired left ventricular function. These relevant comorbidities and application of rapid ventricular pacing result in a high risk for hemodynamic deterioration during valve implantation with potentially life-threatening situations. Lifesaving actions might be associated with an inherent risk for adverse events. Venoarterial extracorporeal membrane oxygenation (ECMO) offers the option for temporary cardiac support and can be implanted in advance when hemodynamic problems are anticipated. In case of sudden hemodynamic collapse, emergency implementation of ECMO support can be instituted during the procedure via peripheral vessels. ${ }^{1}$

received

November 28, 2015

accepted after revision

January 13, 2016

published online

February 24, 2016

\section{Case Description}

A 71-year-old man with severe aortic valve stenosis was planned for TAVI due to a reduced clinical condition and the presence of relevant comorbidities including reduced left ventricular ejection fraction of $30 \%$, coronary artery disease, chronic obstructive pulmonary disease, kidney insufficiency, and history of abdominal surgery for colon carcinoma. After routine preoperative workup, a transfemoral implantation of a CoreValve $(29 \mathrm{~mm}$, Medtronic, Minneapolis, Minnesota, United States) was planned in general anesthesia. At our institution, transfemoral TAVIs are routinely executed in general anesthesia to facilitate transesophageal echocardiography for intraprocedural guidance and postprocedural quality control. A 6-French $(F)$ pigtail catheter was inserted via the left common femoral artery for contrast media visualization of the aortic root, a pacemaker wire was inserted via the left
License terms

Stuttgart · New York

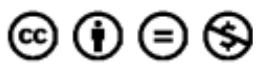


femoral vein for rapid ventricular pacing, and an 18-F sheath was inserted via the right common femoral artery for balloon valvuloplasty and valve implantation. After unproblematic balloon valvuloplasty, the CoreValve was advanced into the desired position. Valve deployment was then started under fluoroscopic guidance; after the release of about two-thirds, the valve threatened to slide into the ventricle with subsequent drop of blood pressure. The valve was retracted into the delivery catheter while cardiopulmonary resuscitation was started. Since the patient could not be stabilized, ECMO cannulas were implanted into the vessels of the left groin using the arterial (18-F cannula) and venous (22-F cannula) access of the previously inserted pigtail catheter and pacemaker wire, respectively. Pericardial effusion and rupture of the aortic root were excluded as potential triggers for the hemodynamic impairment while the patient was stabilized under ECMO support. However, a pigtail catheter was no more present for the injection of contrast media to guide the unfinished CoreValve implantation. Therefore, a Y-connector (3/8"-3/8"-1/4") was plugged into the arterial line of the ECMO. An additional 18-F sheath was shortened and inserted into the $1 / 4^{\prime \prime}$ connector with subsequent deairing (-Figs. 1 and 2). This sheath facilitated the introduction of a pigtail catheter for contrast media-guided and uneventful implantation of the CoreValve prosthesis.

After 30 minutes of reperfusion, the ECMO was successfully weaned, all insertion sites were manually compressed to avoid further bleeding and the patient was transferred to the intensive care unit. The patient's stay in the intensive care unit was 7 days and finally was discharged after additional 22 days.

\section{Discussion}

In the present case report, we describe the advancement of a pigtail catheter through an arterial extracorporeal membrane

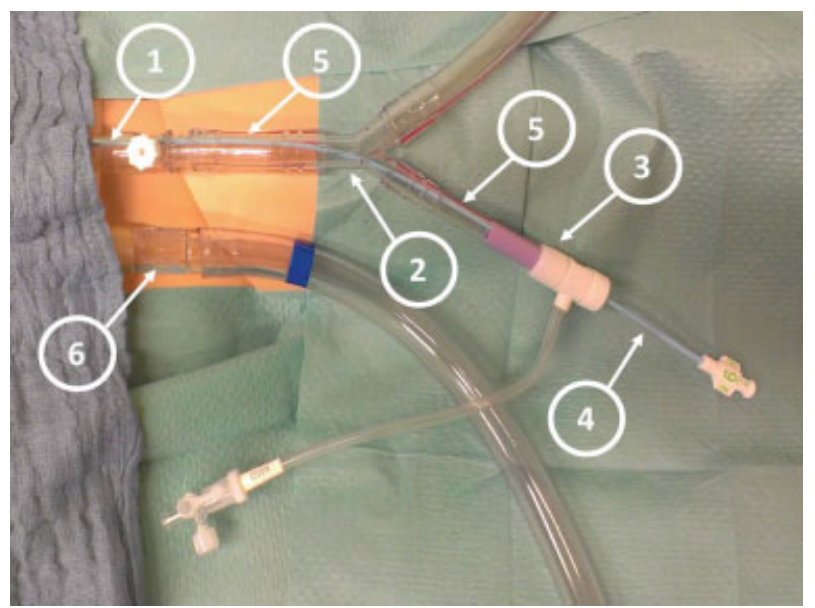

Fig. 1 Dummy model of intraoperative setting with arterial and venous extracorporeal membrane oxygenation cannulas. A Y-connector (2) (3/8"-3/ $8^{\prime \prime}-1 / 4^{\prime \prime}$ ) was plugged into the arterial line (1) and an 18-F sheath (3) was joined to the $1 / 4^{\prime \prime}$ connector using a connecting tube (5) facilitating the insertion of a pigtail catheter (4). Venous line (6).

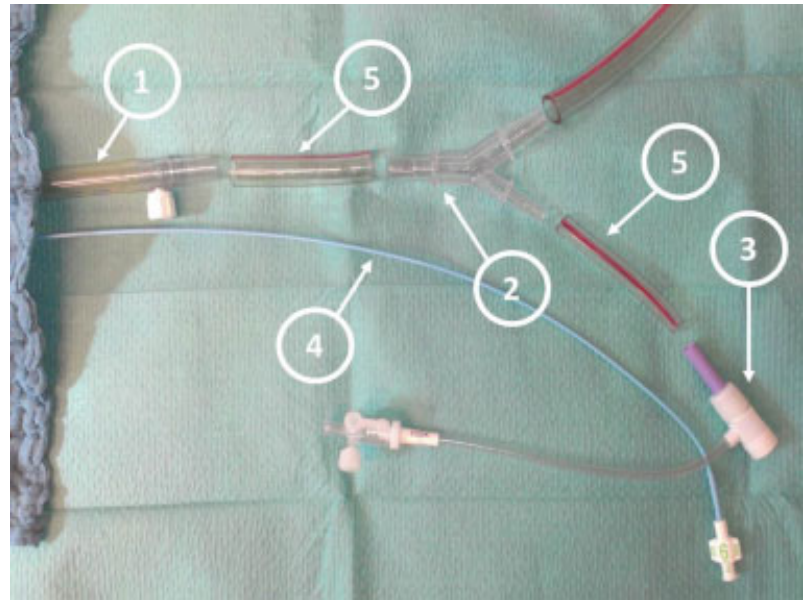

Fig. 2 Dummy model of all implemented and separated parts used for the arterial line. Arterial cannula (1); Y-connector (2); 18-F sheath (3); pigtail catheter (4); and connecting tubes (5).

oxygenator cannula during a TAVI procedure. Insertion of a Yconnector facilitated injection of contrast media into the aortic root for exact positioning of the CoreValve after emergency establishment of ECMO support due to hemodynamic collapse. Of note, the additional insertion of the pigtail catheter through the 18-F arterial cannula did not markedly impair the ECMO flow. Additional distal limb perfusion was not instituted after ECMO implantation since we decided to restrict the use of extracorporeal circulation on the time in the hybrid operating room. Luckily, the patient could be weaned from the ECMO in the operating room and was transferred to the intensive care unit.

The EndoReturn Arterial Cannula (Edwards Lifesciences Co., Irvine, California, United States) would have been an alternative to the custom-made additional port for the pigtail catheter as described in the presented case. This cannula features a "side arm" with a hemostasis valve that allows the passage of additional catheters. However, our routine cannula for peripheral ECMO implantation was used in the emergency situation. Additional costs would incur in routine use of the EndoReturn Arterial Cannula.

As we described an urgent ECMO implantation due to sudden hemodynamic collapse, Arlt et al reported about a series of four TAVI patients with cardiopulmonary resuscitation or hemodynamic instability with need for ECMO support, respectively. In two patients, the transcatheter procedure was successfully continued; two further patients were transferred for surgical aortic valve replacement of which one patient died 6 days after the procedure due to multiorgan failure. ${ }^{2}$

ECMO support can also be instituted before beginning a TAVI procedure in case of conceivable hemodynamic problems. Seco et al reported about eight patients with prophylactic ECMO implantation in patients undergoing TAVI procedures. The decision for ECMO implementation was made by the heart team due to hemodynamic instability after balloon aortic valvuloplasty, general "borderline hemodynamics" before the definitive valve implantation, valve malpositioning, or ventricular fibrillation. The authors finally present one patient with a life-threatening bleeding but no mortality. ${ }^{3}$ 
In summary, the presented case report describes a safe technique of pigtail insertion via an arterial ECMO cannula for contrast media injection during TAVI and can help successfully finish TAVI procedures even after unplanned ECMO support.

\section{Note}

This work was presented during the Sixth "Fokustagung Herz" in Nuremberg in November, 2014.

\section{References}

1 Beckmann A, Benk C, Beyersdorf F, et al. ECLS Working Group. Position article for the use of extracorporeal life support in adult patients. Eur J Cardiothorac Surg 2011;40(3):676-680

2 Arlt M, Philipp A, Voelkel S, et al. Early experiences with miniaturized extracorporeal life-support in the catheterization laboratory. Eur J Cardiothorac Surg 2012;42(5):858-863

3 Seco M, Forrest P, Jackson SA, et al. Extracorporeal membrane oxygenation for very high-risk transcatheter aortic valve implantation. Heart Lung Circ 2014;23(10):957-962 